\title{
A systematic review and meta-analysis comparing the effect of aquatic and land exercise on dynamic balance in older adults
}

\author{
Youngwook Kim ${ }^{1 *}$ D, Michael N. Vakula', Benjamin Waller ${ }^{2}$ and Eadric Bressel ${ }^{1}$
}

\begin{abstract}
Background: Balance impairments are the leading causes of falls in older adults. Aquatic-based exercises have been broadly practiced as an alternative to land-based exercises; however, the effects on dynamic balance have not been comprehensively reviewed and compared to land exercises. Thus, the purpose of this systematic review and meta-analysis was to compare the effectiveness of aquatic exercises (AE) to land exercises (LE) on dynamic balance in older adults.

Methods: Electronic databases (PubMed, MEDLINE, CINAHL, SPORTDiscus, psycINFO), from inception to November 2019, were searched. Studies met the following eligibility criteria: Randomized controlled trials, English language, older adults aged 65 years or older, a minimum of one AE and LE group, at least one assessment for dynamic balance. For the meta-analysis, the effect sizes of dynamic balance outcomes were calculated using a standardized mean difference (SMD) and a 95\% confidence interval (CI).

Results: A total of 11 trials met the inclusion criteria, and 10 studies were eligible for the meta-analysis. The metaanalysis presented that older adults in AE groups demonstrated comparable enhancements in dynamic steady-state balance (SMD $=-0.24 ; 95 \% \mathrm{Cl},-.81$ to .34$)$, proactive balance $(\mathrm{SMD}=-0.21 ; 95 \% \mathrm{Cl},-.59$ to .17$)$, and balance test batteries (SMD $=-0.24 ; 95 \% \mathrm{Cl},-.50$ to .03$)$ compared with those in LE groups.

Conclusions: AE and LE have comparable impacts on dynamic balance in older adults aged 65 years or older. Thus, this review provides evidence that $A E$ can be utilized as a reasonable alternative to LE to improve dynamic balance and possibly reduce the risk of falls.
\end{abstract}

Keywords: Older adults, Seniors, Aquatic exercise, Aquatic therapy, Balance, Dynamic balance, Falls, Fall prevention

\section{Background}

In adults aged 65 years or older, approximately $29 \%$ of the population experience at least one fall per year, and the rate of falls and fall-related injuries increase with age [1]. Falls are a common cause of morbidity and mortality

\footnotetext{
* Correspondence: youngwook.kim@aggiemail.usu.edu

'Department of Kinesiology and Health Science, Utah State University, 7000 Old Main Hill, Logan, UT 84322-7000, USA

Full list of author information is available at the end of the article
}

including both fatal and non-fatal injuries and poor quality of life [2, 3]. Falls often cause substantial medical costs. In 2015, fatal fall-related and non-fatal fall-related injuries cost an estimated \$637.5 million and \$31.3 billion, respectively [4]. Considering the globally increasing proportion of older adults, the medical costs related to falls may constantly increase unless cost-effective interventions are established and implemented.

C C The Author(s). 2020 Open Access This article is licensed under a Creative Commons Attribution 4.0 International License, which permits use, sharing, adaptation, distribution and reproduction in any medium or format, as long as you give appropriate credit to the original author(s) and the source, provide a link to the Creative Commons licence, and indicate if changes were made. The images or other third party material in this article are included in the article's Creative Commons licence, unless indicated otherwise in a credit line to the material. If material is not included in the article's Creative Commons licence and your intended use is not permitted by statutory regulation or exceeds the permitted use, you will need to obtain permission directly from the copyright holder. To view a copy of this licence, visit http://creativecommons.org/licenses/by/4.0/ The Creative Commons Public Domain Dedication waiver (http://creativecommons.org/publicdomain/zero/1.0/) applies to the data made available in this article, unless otherwise stated in a credit line to the data. 
Exercise interventions have been effective at improving balance and reducing fall risks in older adults [5-8]. A Cochrane systematic review by Howe et al. indicated that exercise on land is the most common form of treatment in older adults to improve balance and reduce fall risk [9]. However, land-based exercises contain a higher rate of extrinsic fall risk factors (e.g., uneven walking surface) when compared to aquatic exercises, which may, in turn, interrupt the progression of a fall prevention exercise program. This is important to note because extrinsic risk factors account for the majority of all falls [10]. These aforementioned limitations associated with the safety issues during land-based exercises are less common in aquatic-based exercise programs [11].

Aquatic exercises have been utilized as an alternative to land-based exercises for older adults that display lower physical activity levels, neuromuscular degeneration, or orthopedic disabilities that affect balance, mobility, and pain [12-14]. For this systematic review and meta-analysis, we defined the aquatic exercise as any type of exercise performed in water. The buoyant force of water and the hydrostatic pressure/density help participants slow the movement, and additional sensory cues supplied by the viscosity of water facilitate muscle recruitment timing [15]. Thus, water provides a safe, low risk, and supportive training environment, which may be advantageous for older adults to participate in exercise programs without the risk or fear of falling [16].

Previous systematic reviews have summarized empirical evidence for aquatic exercises on strength, mobility, flexibility, balance, and various health outcomes in older adults $[12,13,17]$. Observations from these reviews have indicated that aquatic exercises may improve the aforementioned outcome measures. Specifically, a recent systematic review and meta-analysis summarized statistical evidence for aquatic exercise on dynamic balance for the first time and reported that aquatic exercise significantly improved dynamic balance in older adults with knee or hip osteoarthritis [18]. However, only four studies and one outcome measure (Timed Up and Go test) were included in the meta-analysis, and the population was limited to osteoarthritic patients. Moreover, the results of aquatic exercise were compared to the controls, thus, evidence regarding the effectiveness of aquatic exercises over comparable land-based exercises in older adults is inconclusive. Due to complex environments continuously challenging older adults, various dynamic balance abilities, that can be defined as the ability to control postural stability while in motion [19], are critical in this population [20]. Accordingly, there is a need to more formally quantify the effects of $\mathrm{AE}$ on dynamic balance concerning fall prevention protocols. This systematic review and meta-analysis aimed to compare the effects of aquatic exercise (AE) and land exercise (LE) on dynamic balance in older adults aged 65 years or older. The PICO question was as follows: "Are aquatic exercises more effective than land-based exercises at improving dynamic balance in older adults aged 65 years or older?"

\section{Methods}

A systematic review of the literature with meta-analysis was conducted in November 2019 to examine the effects of $\mathrm{AE}$ on dynamic balance in older adults. The following electronic databases were searched by one reviewer (Y.K.) on November 19th, 2019: PubMed (1965-), MEDL INE (1959-), CINAHL (1984-), SPORTDiscus (1978-), psycINFO (1958-). The databases were examined using the following combination of keywords: (aquatic therapy OR aquatic activity OR aquatic aerobics OR aquaerobics OR aquatic exercise OR aquatic physical therapy OR aquatic physiotherapy OR aquatic rehabilitation OR hydrotherapy OR pool exercise* OR pool therapy OR swimming OR swimming therapy OR water aerobics OR water-based exercise OR water exercise OR water rehabilitation OR water therapy OR water rehabilitation OR water activity, OR water sport*) AND (aged OR older OR elderly OR senior) AND (balance OR postur*). There was no restriction on the publication year.

All articles identified in the database search were exported to Zotero 5.0.66 (http://www.zotero.org) and any duplicates were deleted. Two reviewers (Y.K. and M.V.) initially screened, included, and excluded studies based on titles and abstracts. Full text of identified articles was obtained and reviewed by the first and second reviewers (Y.K. and M.V.). Disagreements were resolved by discussion and third (E.B.) and fourth (B.W.) reviewers were consulted as necessary. This systematic review and meta-analysis was prospectively registered in the Open Science Framework (OSF). The OSF registration number was 9bc4y. Protocol details can be accessed via https://osf.io/9bc4y.

\section{Eligibility criteria Type of participants}

Studies that recruited adults aged 65 years or older were included. There was no restriction on the injury or disorder type, settings, and the history of falls. Animal studies and human studies with participants aged under 65 were excluded.

\section{Type of studies}

Studies conducted as a randomized control trial (RCT) and published in the English language were considered for inclusion. Studies with other research designs or non-peer-reviewed articles were excluded. 


\section{Intervention}

Studies that employed all types of AE with a description of intervention details, such as duration, frequency, type, and intensity of AE, were included. The studies must have included a minimum of one $\mathrm{AE}$ group and a comparison group participating in another exercise program on dry land. Studies that did not include exercise components, such as bath or spa therapies, were excluded.

\section{Outcome measures}

Studies must have reported at least one outcome related to dynamic balance and compared the outcomes between AE and LE groups. All outcome measures must have been conducted on land because postural adjustment and movement patterns are significantly altered in water [21-23], and daily living activities are mostly performed on dry land. Studies including mixed intervention (e.g., both AE and LE in all groups) were excluded and any studies not providing data on the baseline or end-point outcomes were additionally excluded from the meta-analysis.

\section{Data extraction and coding}

A total of 11 studies meeting the eligibility criteria were reviewed and coded in REDCap (https://www.projectredcap.org/). All relevant information was extracted for each study as follows: (1) report characteristics (2) participants (3) AE settings (4) interventions (5) outcome measures (6) results. The included studies were assessed and coded independently by two reviewers (Y.K. and M.V.) and discussed for consensus. If there was a disagreement, the study was re-evaluated to achieve consensus.

\section{Risk of bias and publication bias assessment}

The analysis of the methodological quality and risk of bias of the included studies was conducted using the Cochrane risk of bias tool ( $\operatorname{RoB} 2)$ [24] independently by two authors (Y.K. and M.V.). The tool can be utilized to assess the impact of each potential source of bias, at the "low", "high", and "somewhat concerns" risk level, respectively. The following criteria that potentially affect the risk of bias were addressed: randomization process, deviation from intended interventions, missing outcome data, measurement of outcome, selection of the reported result, and overall bias. Any disagreements were discussed until consensus was reached and additionally arbitrated by the third (E.B.) and fourth (B.W.) reviewers if needed. "Small study effects" is a generic term for the phenomenon that smaller studies sometimes show different, often larger, treatment effects than large studies [25]. In meta-analysis, small study effects are a wellknown challenging and critical issue that may threaten the validity of the study results, and the most well- known reason of the small study effects is publication bias [25]. The publication bias can be displayed graphically in funnel plots, thus, a small study effect was examined and interpreted through a test for funnel plot asymmetry [26]. In the absence of publication bias, the plot should be shaped like a symmetrical funnel with small studies scattered widely at the bottom of the graph and larger studies spread narrowly [25].

\section{Meta-analysis}

The purpose of the meta-analyses was to compare the pooled effect size between the AE group and LE group on dynamic balance in older adults. For the postintervention sample size, when all subjects at the baseline were followed up, assessed, and analyzed regardless of their compliance to the intervention (intention-totreat), the data including means and standard deviations for each outcome measure were used on the preferential basis [27]. Otherwise, the data of subjects who completed a pre-determined intervention(s) and have measurable data at the primary end point without any major protocol violations (per protocol) were used [27]. When data were not reported in the article as means and standard deviations, we contacted the corresponding authors and requested the data.

Outcome measurements included in the meta-analysis were assigned into three categories: (a) dynamic steadystate balance (e.g., 5-m walk test, 10-m walk test, backward tandem walk), (b) proactive balance (e.g., FRT; Functional Reach Test, TUG; Timed Up and Go test, 8$\mathrm{ft}$ up-and-go test), and (c) balance test batteries (e.g., BBS; Balance Berg Scale and BOOMER; Balance Outcome Measure for Elder Rehabilitation) [28]. Where a trial reported more than one outcome in one of these categories, only one outcome with the highest priority was used for the analysis in line with Lesinski et al. [29]. The highest priority was given to the gait speed in the dynamic steady-state balance, FRT in the proactive balance, and BBS in the balance test battery [29]. When these representative outcomes were not available, the most similar outcomes related to the temporal (duration) and spatial (form of the motion) structure were used [29]. For a crossover RCT study [30], first-phase data were used. Sensitivity analyses were additionally performed to explore the robustness of the results by quantifying the differences in outcomes when removing one trial with a distinctly different direction of change in each category of balance outcome measurements.

The effect sizes between AE and LE groups were described as standardized mean differences (SMD) and 95\% confidence intervals (CI). An effect size (SMD) 0.2$0.5,0.5-0.8$, and $>0.8$ were considered a small, moderate, and large effect, respectively [31]. In case of a lower score indicating better performance in dynamic balance, 
scale directions were adjusted by multiplying -1 to data, which resulted in a positive value indicating an improvement in favor of AE. For all analyses, we used an inverse-variance weighted random-effects model. All meta-analyses were performed using the Cochrane Collaboration's Review Manager Software (RevMan 5.3.).

\section{Results}

\section{Study selection}

The electronic search retrieved a total of 2969 potential studies in the five databases, and no additional studies were identified by hand searching. Of these studies, 1491 duplicates were removed, and 1445 studies were excluded based on title and abstract content. We obtained the full text of the remaining 33 trials, 22 of which were excluded because they did not meet eligibility criteria. Finally, 11 studies were retained for our systematic review, and 10 studies were included in the meta-analysis after excluding one study due to insufficient data [32]. The flow diagram in Fig. 1 schematizes the steps of the selection of the studies.

\section{Characteristics of included studies \\ Participants}

Eleven studies included in this systematic review were randomized controlled trials, which compared the impacts of $\mathrm{AE}$ and $\mathrm{LE}$ on dynamic balance in older adults aged 65 years or older. Table 1 presents the characteristics of participants of the 11 eligible studies that provided data for 372 participants with the mean age of $69.6 \pm 4.0$ years. The participants were recruited from community [11, 32, 33], hospital [30, 34, 35], and Parkinson's associations [36-38]. Attrition rates were calculated using the following formula: Number of participants lost at post-intervention/number of participants at baseline ${ }^{*} 100$. The attrition rates ranged from 0 to $27 \%$.

\section{Aquatic setting and interventions}

First, focusing on the pool characteristics, 10 studies reported the type of pool where the AE took place: Five at indoor swimming pools, three at therapeutic pools, two at outdoor swimming pools, and one not reported. The water depth varied from $1 \mathrm{~m}$ to $1.8 \mathrm{~m}$, and the water

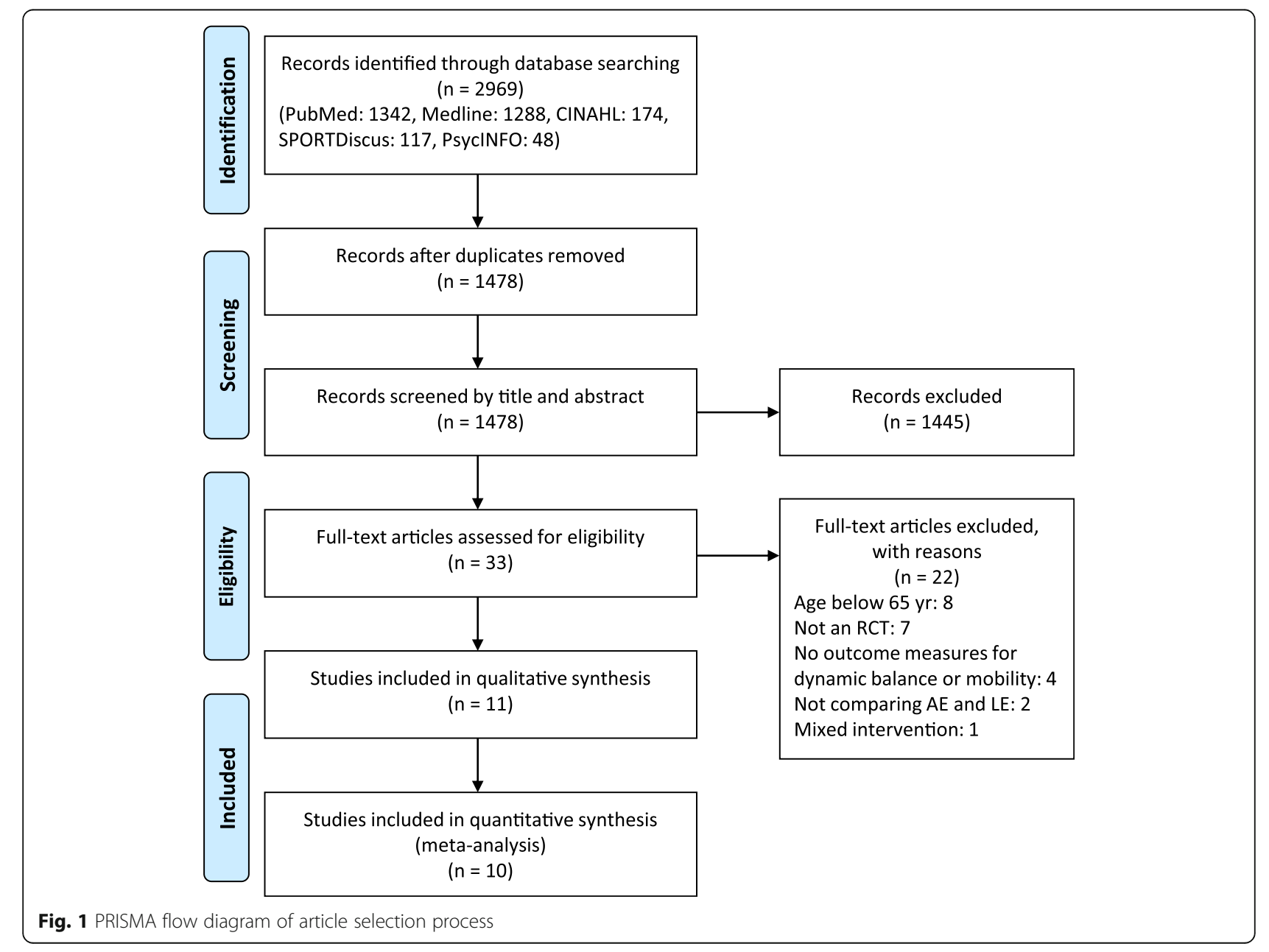




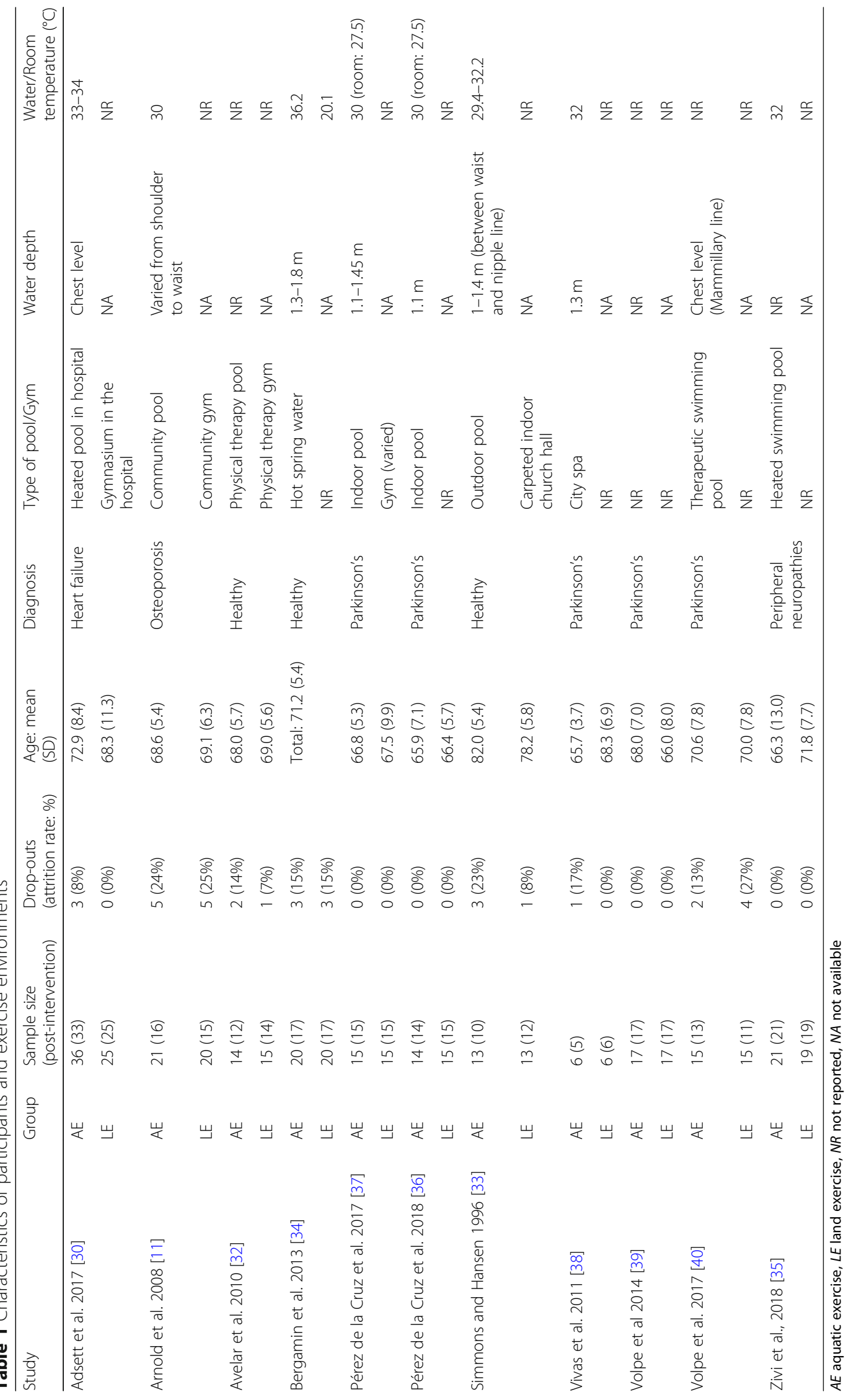


temperature ranged between $27.5^{\circ} \mathrm{C}$ and $36.2^{\circ} \mathrm{C}(31.5 \pm$ $2.6^{\circ} \mathrm{C}$ ) with an exception of three studies not reporting the aquatic setting [32, 39, 40]. The characteristics of pools are reported in Table 1.

The AE programs exhibited substantial differences across all included studies in regards to the intervention duration (45-60 min), frequency (1-5 sessions per week), and total duration (4-20 weeks) (Table 2). The AE programs identified included gait, mobility, stretching, stabilization, resistance, balance, endurance, strengthening, aerobic training, and Ai Chi. The exercises provided for AE and LE groups had the same or similar types, volume, emphasis, and objectives, except for two studies $[36,37]$. Table 2 presents a summary of the exercise programs.

\section{Outcome measurements and summary of the results}

All studies included in this review performed at least one dynamic balance-related measurement before and after the intervention on land. Four studies evaluated long-term effects at additional stages after the intervention was terminated [36-38, 40], but the second postintervention outcome measure data were not used due to differences in the time points after interventions and limited data. Overall, eight studies reported greater improvements in AE groups compared to LE groups in at least one dynamic balance outcome measurement [11, 33-39], whereas two studies did not find any statistically significant differences between $\mathrm{AE}$ and LE groups [32, 40], and one study reported a greater improvement in LE group in one outcome measurement [30]. Table 3 presents the details of outcome measurements and a brief summary of the results of individual studies.

\section{Risk of bias and publication bias}

The Cochrane risk of bias tool indicated a "low" risk of bias for two studies [35, 37] and "high" risk of bias for four studies [33, 34, 38, 40] due to randomization process [33] and missing outcome data [33, 34, 38, 40]. The other five studies had "somewhat concerns" [11, 30 , $32,36,39]$ due to randomization process [32] and selection of the reported result [11, 30, 32, 36, 39]. Figure 2 presents the risk of bias of the included studies. The visual inspection of the funnel plot identified substantial asymmetry, indicating the possibility of publication bias in the meta-analysis (Fig. 3).

\section{Meta-analysis}

Post-intervention assessment data for BBS, Dynamic Gait Index, tandem gait, and $10 \mathrm{~m}$ gait speed from the study by Avelar et al. [32], data for 5-m walk test, FRT, and TUG from the study by Vivas et al. [38], data for BBS from the study by Arnold et al. [11], and data for $10-\mathrm{m}$ gait speed and BOOMER from the study by Adsett et al. [30] were requested, and all data, except those from the study by Avelar et al. were received. Thus, a total of 10 studies were included in the meta-analysis of dynamic balance outcomes for AE compared with LE [11, 30, 33-40].

Outcome measurements included in each category were as follows: (a) dynamic steady-state balance: 10-m walk test (speed) [30], 5-m walk test (speed) [38], and backward tandem walk (number of errors) [11], (b) proactive balance: FRT [11, 33, 38, 39], TUG [30, 36, 37, 40], and 8-ft up-and-go test [34], (c) balance test batteries: BBS [11, 35, 37-40] and BOOMER [30]. When a random-effect analysis was applied using the 10 studies involving 343 participants, AE groups compared with LE groups displayed comparable improvements in dynamic steady-state balance (SMD $=-0.24 ; 95 \% \mathrm{CI},-.81$ to .34 ), proactive balance $(\mathrm{SMD}=-0.21 ; 95 \% \mathrm{CI},-.59$ to .17 ), and balance test batteries $(\mathrm{SMD}=-0.24 ; 95 \% \mathrm{CI},-.50$ to .03) (Fig. 4). The sensitivity analyses after excluding one trial with a distinctly opposite direction of change in each category presented that the point estimates changed by -0.20 ( $\mathrm{SMD}=-0.44 ; 95 \% \mathrm{CI},-.88$ to 0$)$ in dynamic steady-state balance, by $-0.08 \quad(\mathrm{SMD}=-0.29$; $95 \% \mathrm{CI},-62$ to .03$)$ in proactive balance, and by -0.08 $(\mathrm{SMD}=-0.32 ; 95 \% \mathrm{CI},-.61$ to -.03$)$ in balance test batteries (Fig. 5).

\section{Discussion}

This is the first systematic review with meta-analysis comparing the effects of $\mathrm{AE}$ and $\mathrm{LE}$ on dynamic balance in older adults. Eight of the included studies [11, 33-39] concluded that $\mathrm{AE}$ resulted in greater improvements in at least one dynamic balance outcome measurement compared to LE. However, the results of the metaanalysis revealed no statistically significant differences in all outcome categories. This result is consistent with a previous review conducted by Waller et al. that compared the effects of aquatic and land-based exercise programs on physical functioning in healthy older adults and demonstrated small effect sizes in postural stability in favor of $\mathrm{AE}$ and in walking ability in favor of LE [13]. In consideration of the limited number of studies included in this analysis and results of the sensitivity analyses, however, the results must be interpreted with caution.

Although different musculoskeletal or neurological disorders do not share identical signs or symptoms, dynamic balance is important across all older populations to prevent fall risk and to enhance rehabilitation from fall-related injuries. For example, Parkinson's disease is a degenerative neurological disorder commonly reported in the senior population, and the risk of falls and fallrelated injuries increase in this population due to deficits in motor functions and postural stability [41]. 


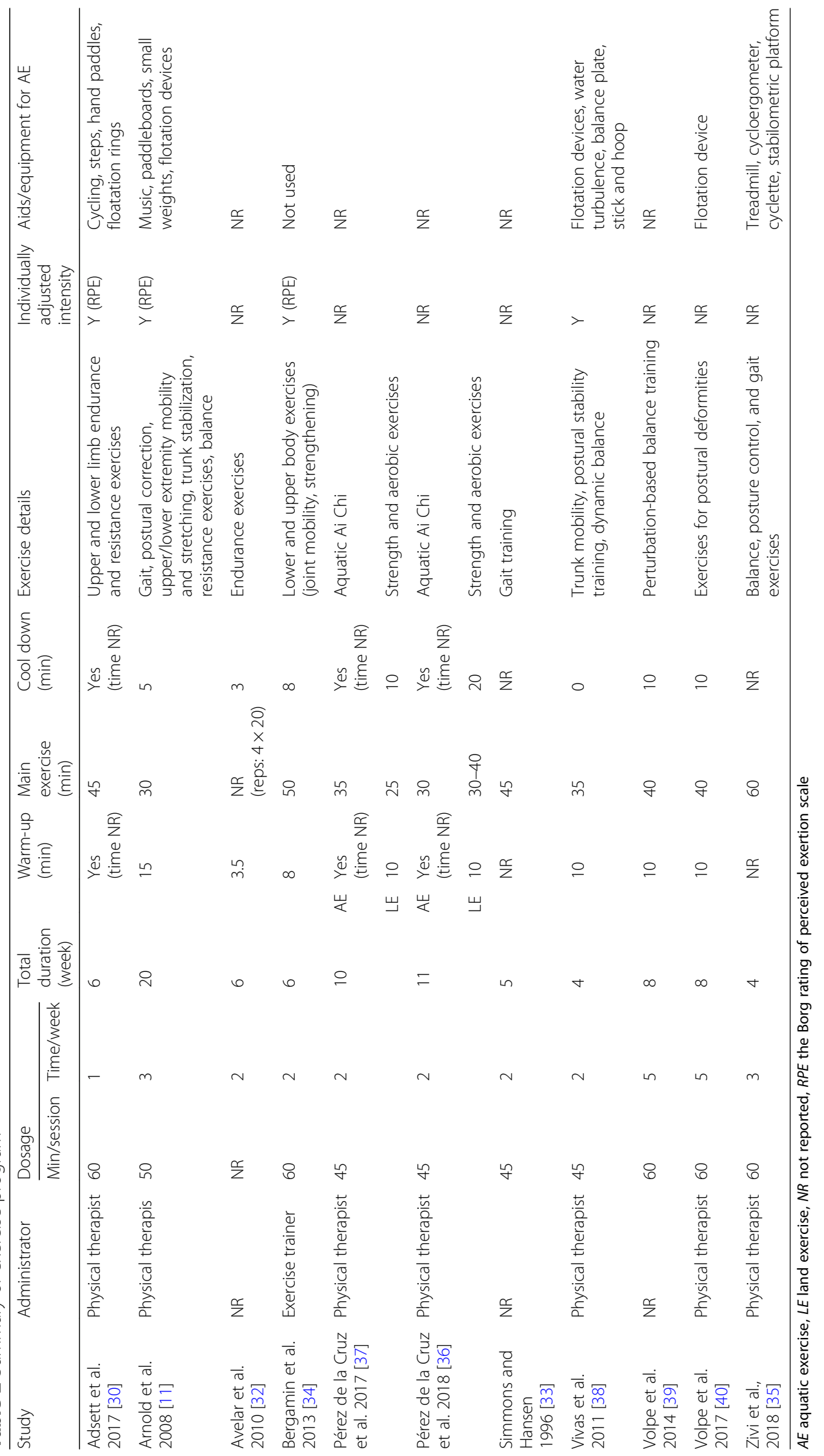


Table 3 Outcome measures and summary of main findings of all selected studies

\begin{tabular}{|c|c|c|c|c|c|}
\hline Study & Outcome measures & Follow-up & Adverse events & $\begin{array}{l}\text { Participants } \\
\text { feedback }\end{array}$ & Results \\
\hline $\begin{array}{l}\text { Adsett et al. } 2017 \\
\text { [30] }\end{array}$ & $\begin{array}{l}\text { 6MWT, TUG, 10-m walk } \\
\text { test (speed), BOOMER }\end{array}$ & $\mathrm{N}$ & $\begin{array}{l}\text { Shortness of breath (1), } \\
\text { dizziness (2) }\end{array}$ & Reported & $\begin{array}{l}\text { LE group showed greater improvements } \\
\text { in 6MWT. No significant differences in } \\
10-\mathrm{m} \text { gait speed and BOOMER. }\end{array}$ \\
\hline $\begin{array}{l}\text { Arnold et al. } 2008 \\
\text { [11] }\end{array}$ & $\begin{array}{l}\text { BBS, FRT, backward } \\
\text { tandem walk }\end{array}$ & $\mathrm{N}$ & $\begin{array}{l}\text { Pain: } 29 \% \text { AE, } 52 \% \text { LE. } \\
\text { Muscle cramping and } \\
\text { stiffness: } 25 \% \text { AE, } 3 \% \text { LE }\end{array}$ & NR & $\begin{array}{l}\text { AE group showed a greater improvement } \\
\text { only in the backward tandem walk versus } \\
\text { LE group. No significant differences in BBS } \\
\text { and FRT between two groups. }\end{array}$ \\
\hline $\begin{array}{l}\text { Avelar et al. } 2010 \\
\text { [32] }\end{array}$ & $\begin{array}{l}\text { DGI, BBS, Tandem gait } \\
\text { test, } 10-m \text { gait speed test }\end{array}$ & $\mathrm{N}$ & NR & $N R$ & $\begin{array}{l}\text { Both intervention groups showed } \\
\text { improvements only in DGI and BBS, with } \\
\text { no difference between groups. }\end{array}$ \\
\hline $\begin{array}{l}\text { Bergamin et al. } \\
2013[34]\end{array}$ & 8-foot up-and-go test & $\mathrm{N}$ & None & $N R$ & $\begin{array}{l}\text { Both intervention groups showed } \\
\text { improvements, with significantly greater } \\
\text { improvement in AE group. }\end{array}$ \\
\hline $\begin{array}{l}\text { Pérez de la Cruz } \\
\text { et al. } 2017 \text { [37] }\end{array}$ & $\begin{array}{l}\text { BBS, Tinetti Scale, } \\
\text { FTSTS, TUG }\end{array}$ & 1 month & None & NR & $\begin{array}{l}\text { Only AE group showed improvements in } \\
\text { all variables, except the FTSTS. LE group } \\
\text { showed no improvements in any of the } \\
\text { balance measures. }\end{array}$ \\
\hline $\begin{array}{l}\text { Pérez de la Cruz } \\
\text { et al. } 2018 \text { [36] }\end{array}$ & TUG, FTSTS, & 1 month & NR & NR & $\begin{array}{l}\text { AE (Ai Chi) group showed improvements } \\
\text { in TUG and FTSTS in post-treatment and } \\
\text { 1-month follow-up, whereas the dryland } \\
\text { group showed no significant differences. }\end{array}$ \\
\hline $\begin{array}{l}\text { Simmons and } \\
\text { Hansen } 1996 \text { [33] }\end{array}$ & FRT & $\begin{array}{l}\mathrm{N} \\
(10-12: \\
\text { injury tracking) }\end{array}$ & NR & NR & $\begin{array}{l}\text { AE group showed gradual improvements in } \\
\text { each week. LE group showed improvement } \\
\text { only in the initial week. At week } 5 \text { (post), AE } \\
\text { group showed significant improvement } \\
\text { compared to LE groups. }\end{array}$ \\
\hline $\begin{array}{l}\text { Vivas et al. } 2011 \\
\text { [38] }\end{array}$ & $\begin{array}{l}\text { FRT, BBS, 5-m walk } \\
\text { test, TUG }\end{array}$ & 17 days & NR & $N R$ & $\begin{array}{l}\text { Both exercise groups showed improvements } \\
\text { in FRT. Only the AE group improved in the } \\
\text { BBS. }\end{array}$ \\
\hline $\begin{array}{l}\text { Volpe et al } 2014 \\
\text { [39] }\end{array}$ & $\begin{array}{l}\text { Instrumental version } \\
\text { of FRT, TUG, BBS, }\end{array}$ & N & None & NR & $\begin{array}{l}\text { Both groups showed improvements in all } \\
\text { outcome variables, with a better improvement } \\
\text { in AE group BBS. }\end{array}$ \\
\hline $\begin{array}{l}\text { Volpe et al. } 2017 \\
\text { [40] }\end{array}$ & TUG, BBS & 2 months & $N R$ & NR & $\begin{array}{l}\text { Both groups showed improvements in all } \\
\text { parameters, with no intergroup differences. }\end{array}$ \\
\hline $\begin{array}{l}\text { Zivi et al., } 2018 \\
\text { [35] }\end{array}$ & BBS, Dynamic Gait Index & $\mathrm{N}$ & NR & NR & $\begin{array}{l}\text { AE group showed a greater improvement in } \\
\text { the Dynamic Gait Index. No significant } \\
\text { difference in BBS between groups. }\end{array}$ \\
\hline
\end{tabular}

Outcome measurements included in the meta-analysis were highlighted (bold), $A E$ aquatic exercise, $L E$ land exercise, $N R$ not reported, $D G I$ Dynamic gait index, $B B S$ Berg Balance Scale, FTSTS Five Times Sit-to-Stand test, TUG Timed Up and Go test, FRT Functional Research Test, 6MWT 6-min walk test, BOOMER Balance Outcome Measure for Elder Rehabilitation

Osteoporosis, which is also common in the senior population, reduces the bone density and results in a higher risk of fractures caused by falling [42]. In addition, those with osteoporosis commonly show muscle weakness, postural deformity, and deteriorated postural control that may significantly increase the risk of falls and fractures $[43,44]$. Thus, various balance abilities have to be trained from both preventive and rehabilitative perspective in those populations. Moreover, dynamic balance is a common interest in all senior populations regardless of the disorder because aging brings a natural biological degeneration in regards to muscle strength and mass and neurological functions [45]. Thus, older adults without any disorder also present a greater risk of falls when compared to younger adults due to inappropriate muscular activation and control of the body's center of mass during ambulation (e.g., dynamic balance) [46]. The comparably effective $\mathrm{AE}$ and $\mathrm{LE}$ in overall older adults suggests that participants can select the training environment based on their preference.

\section{Intervention and outcomes}

Postural strategies vary in different environments regardless of age and physical fitness [16]. Both older and younger adult populations demonstrated the greatest postural sway and sway velocity with the lowest perceived stability in chest-deep water compared to the same measures made at shallow water depths and on land $[16,22,47]$. However, none of the trials included in this current review provided a rationale for the water 


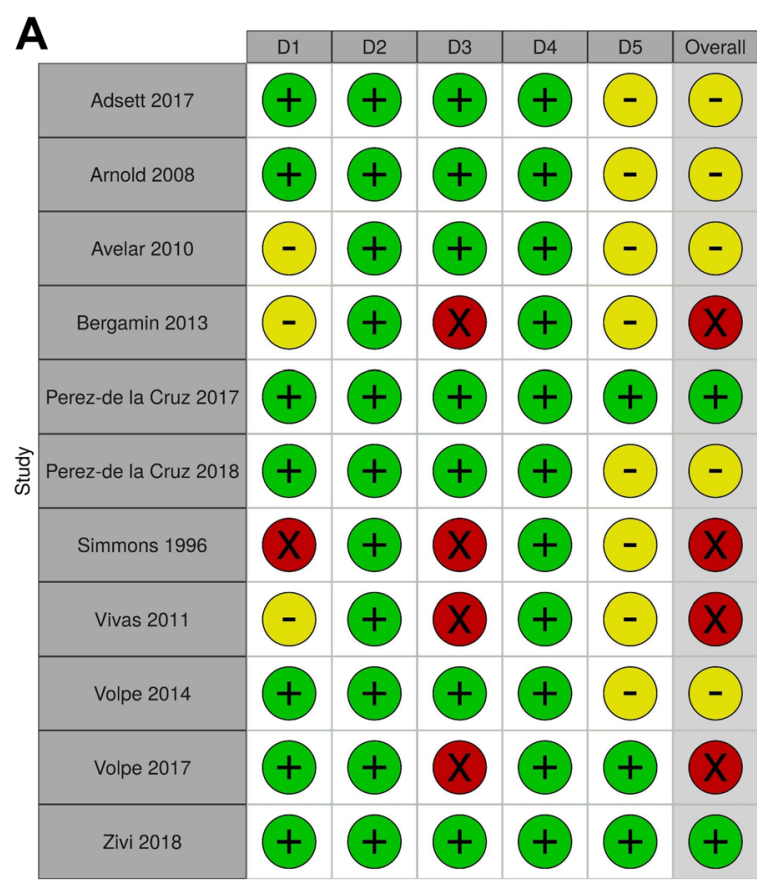

\section{B}

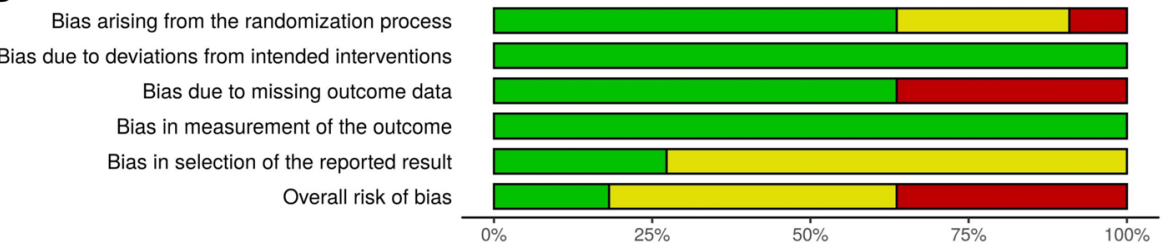

Fig. 2 Risk of bias of the included studies. a Risk of bias graph, b Risk of bias summary. Green, low risk; yellow, somewhat concerns; red, high risk. D1, Randomization process; D2, Deviation from intended interventions; D3, missing outcome data; D4, measurement of outcome; D5, selection of the reported result; Overall, overall bias

depth chosen and considered each participant's height. Although all studies recruited both male and female participants with different mean height, except for only one trial by Arnold et al. [11], the AEs were conducted in water with the non-adjustable water level. That implies the participants in the AE groups were trained with all different exercise intensities despite the identical location, settings, and exercise types. In addition, movement patterns and mechanical power outputs during the same physical performance are presented differently in water and on land [48]. Thus, although most of the trials included provided the same or similar exercise programs to both $\mathrm{AE}$ and LE groups, the subjective exercise intensities can be different due to the environmental factors, which may affect the ultimate training effects. The main reason $\mathrm{AE}$ is recommended to the older adults is to utilize the physical properties of water and provide an optimized medium for exercise. Therefore, it is recommended that future studies provide rationales for water depth and exercise intensities in all intervention groups to investigate and compare the effects between $\mathrm{AE}$ and LE more accurately.

The intervention dose, duration, intensity, and type of exercise varied considerably in each trial, but there was no justification for the exercise dose chosen. According to 'The 2018 Department of Health and Human Services' guideline [49], older adults should get at least 150 min per week of moderate-intensity or $75 \mathrm{~min}$ per week of vigorous-intensity aerobic activity with moderate or high-intensity muscle-strengthening activities at least 2 days a week. Specifically, it is recommended for older adults with the risk of falls to participate in balance training three or more times per week to reduce falls. Older adults in three trials participated in $\mathrm{AE}$ and $\mathrm{LE}$ at least 150 min per week [11, 39, 40], and those in two trials practiced balance training at least 3 times per week $[11,39]$. The intensity of the activities can be perceived in different ways according to various factors, such as physical fitness, muscular performance, or level of disorder or degeneration. Only two studies [11, 34] assessed 


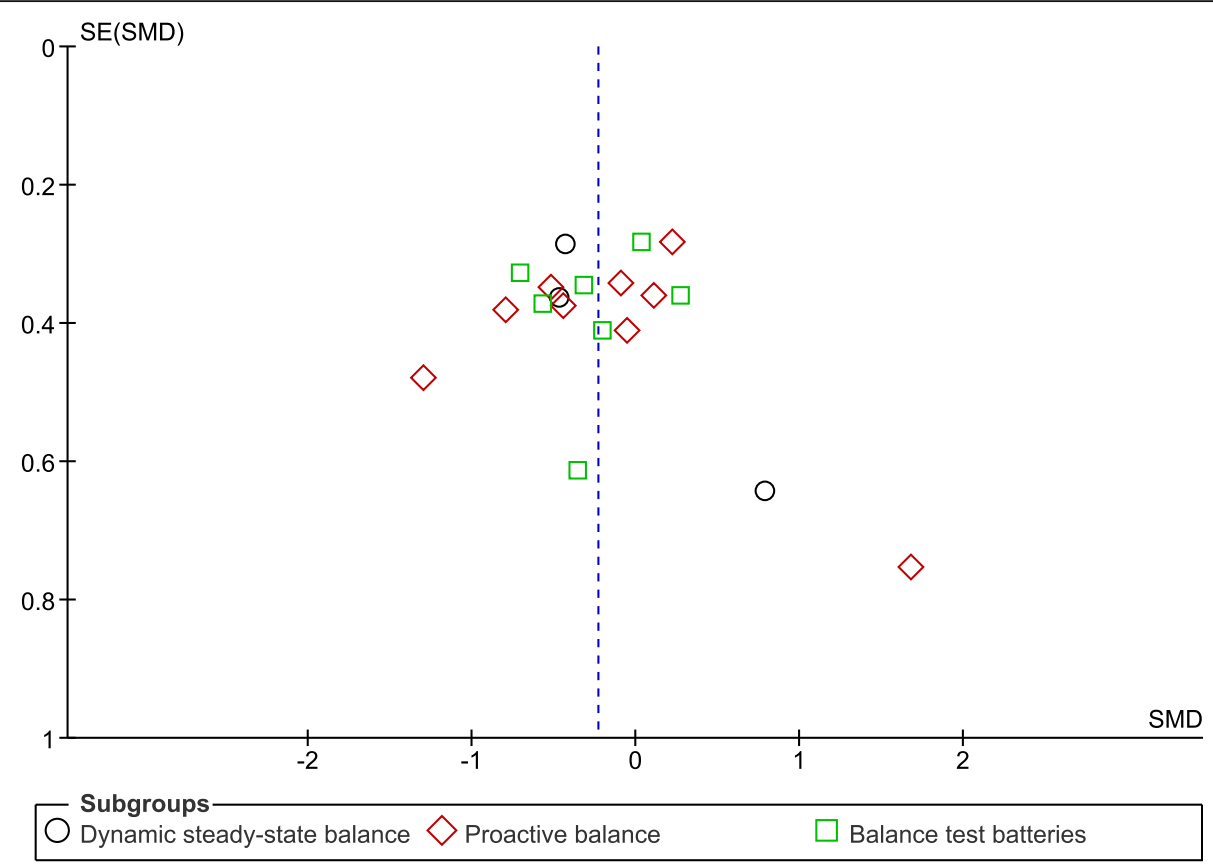

Fig. 3 Funnel plot for all of the meta-analyses

subjective exercise intensity using the Borg rating of perceived exertion scale (RPE scale), and participants were instructed to exercise at a predetermined intensity. However, the optimal dosage, duration, and intensity of $\mathrm{AE}$ were not identified as most of the studies demonstrated low-to-moderate effect sizes and both AE and LE groups mostly presented comparable results across all trials.

The outcomes were measured using various dynamic balance tests, but the assessments were performed immediately after the interventions were terminated. Although each measurement contains critical components in daily living activities and indirectly predicts the potential risk of falls, the generalization of the results regarding the reduction of fall risks must be interpreted with caution as these are lacking in regards to the longer effects of the interventions. Therefore, future studies may wish to evaluate dynamic balance in an extended length of time to assess endurance-related muscle functions that are also essential for postural adjustment in daily life. The aim of $\mathrm{AE}$ interventions in the older population is to improve physical fitness, functional performance, and postural adjustment to ultimately reduce the risk of falls and fall-related injuries and improve their quality of life. Simmons and Hansen [33] tracked the rate of injuries between 10 and 12 months after the termination of the last session and reported that there were no orthopedic injuries from falls in the $\mathrm{AE}$ group, whereas there were two bone fractures (16.7\%) in the LE group since the last session. Two trials conducted by Pérez de la Cruz et al. [36, 37] also included second post- intervention assessments, but the time interval (1-month post-intervention) was not sufficient to determine long term effects of $\mathrm{AE}$ on dynamic balance or fall reductions. Arnold et al. [11] and Volpe et al. [39] reported adverse events that occurred during the interventions, but none of the included studies reported participants' feedback for the AE or LE programs. Besides the main outcome measures, supplementary information regarding injuries and psychological effects, such as satisfaction and enjoyment, may be helpful for an in-depth interpretation of the effectiveness of AE.

In consideration of the exercise program components, the results of the meta-analyses that demonstrated $\mathrm{AE}$ and $\mathrm{LE}$ have equivalent effects on dynamic balance should be interpreted with caution. In general, to improve a specific skill, a completely or nearly identical task is generally included in exercise interventions to induce a practice effect. However, among the ten trials in the meta-analyses, only four trials included at least one balance or gait-related task in the exercise programs $[11,35,38,39]$, and the rest of the ten trials included other types of exercises, such as endurance, strength, mobility, or aerobic exercises, that may contribute to the improvement of dynamic balance. Thus, future research may wish to include a goal-focused exercise program that focuses on balancerelated tasks and controls for other variables, such as exercise intensity, to more clearly compare the effectiveness of $\mathrm{AE}$ and $\mathrm{LE}$ on dynamic balance in the older population. 


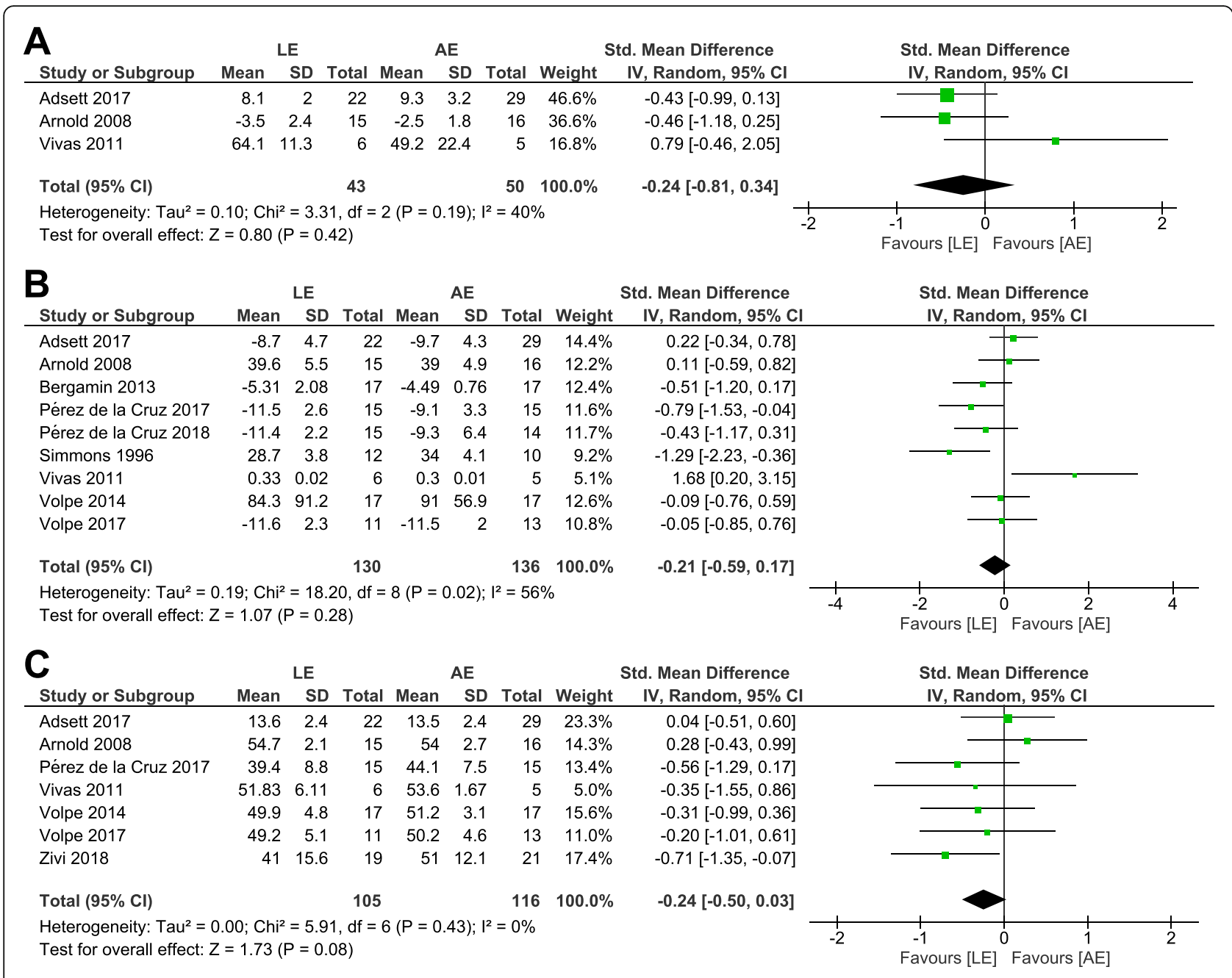

Fig. 4 Forest plot of comparison: AE versus LE. a Dynamic steady-state balance, $\mathbf{b}$ Proactive balance, $\mathbf{c}$ Balance test batteries

\section{Clinical implication}

This study did not identify the statistical superiority of AE over LE programs on dynamic balance. However, these results imply that $\mathrm{AE}$ can be an appropriate alternative to LE which leads to clinically meaningful improvements in balance. Both $\mathrm{AE}$ and LE have different advantages. Because LE is performed under dryland conditions and is more associated with activities of daily living, these can be more applicable and transferable to enable older adults to successfully improve practical skills. Due to environmental characteristics, muscle activation patterns and movement kinematics are different during aquatic activities compared to those during identical land activities $[23,50]$, which may lead to less transferability to various functional tasks on dry land, however, this has not been formally tested or observed in previous research. The aquatic environment provides older adults with numerous biological, neurological, and musculoskeletal advantages and helps them perform higher exercise intensities in a safer and supportive training environment without the risk or fear of falling $[14,48,51-54]$. Therefore, it is suggested that future studies and practitioners select the proper exercise mode that matches each participant's preference and aim of the intervention to maximize the intervention effectiveness. Further investigations regarding the classification of disorder, disease, or history of falls may provide stronger scientific rationales for future balance training protocols for older adults.

As identified in this review, most of the AE programs were administered by physical therapists in clinical facilities. Because of the limited accessibility of aquatic exercise facilities, availability of experts, and higher medical costs, AEs are not broadly practiced in the senior populations. Thus, more easily accessible and lower-cost AE protocols need to be established so that older adults can participate in various physical activities in a safer environment to improve balance, reduce the risk of falls, and ultimately improve their quality of life. 


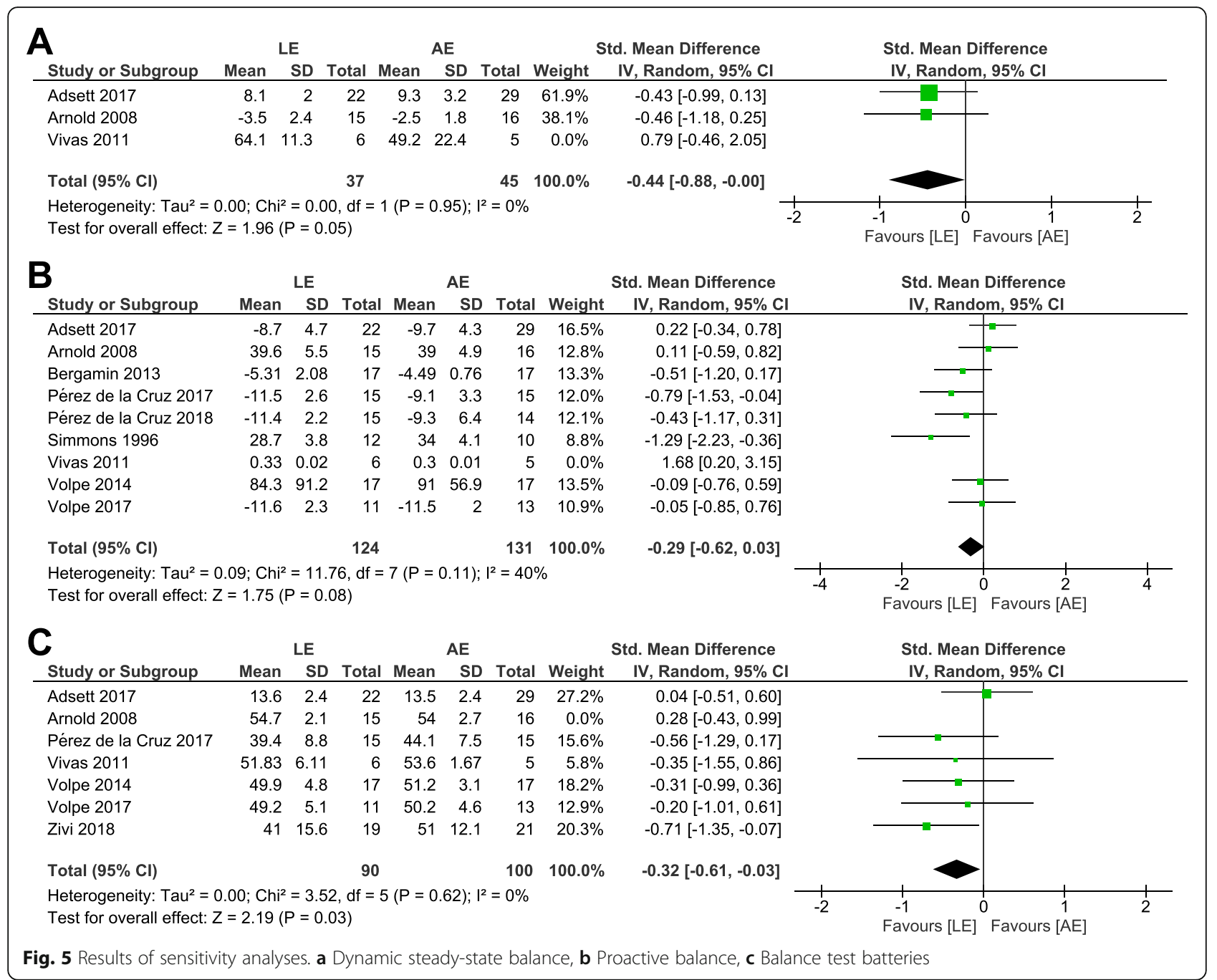

\section{Study limitations}

This systematic review and meta-analysis have several limitations. First, this study was limited to peer-reviewed journal articles published in English and RCT designs only, which may increase the risk of publication bias and potentially exclude appropriate studies with high-quality methodologies. In consideration of the potential small study effects and publication bias, future meta-analyses may want to identify and include unpublished outcomes and unpublished studies to improve the validity of results [55]. Also, we included outcomes using the balance categories instead of using just one measure from each study because we only had 10 studies. Due to the small number of studies included in each category, potential covariates, such as the duration of intervention, exercise type, or exercise intensity, could not be appraised using a moderator analysis. In future reviews, it may be appropriate to use a single measure in each study and conduct a metaregression to identify the impacts of the potential covariates on the effect sizes in the meta-analyses. In addition, five out of 11 studies in the review presented "somewhat concerns" of risk of bias and four had a "high" risk bias, that potentially cause overestimation of the true effects of $\mathrm{AE}$ and $\mathrm{LE}$. The randomization process, missing outcome data, and selection of the reported result were the main causes of bias. Thus, we suggest that future trials make advanced plans for these three categories. Furthermore, as only two outcomes $[33,38]$ in the proactive balance category demonstrated high effect sizes, we were not able to establish the general guideline with optimal exercise type, intensity, dosage, and duration to improve dynamic balance in older adults.

\section{Conclusion}

To summarize, AE displays comparable effects on dynamic balance in older adults aged 65 years or older when compared to LE. Thus, AE may be effectively utilized as a safer alternative to LE, but the results should 
be interpreted with caution due to the limited quantity and risk of bias of the studies. Considering clinical applications, further trials with longer-term outcome measures are needed to elucidate effective AE protocols on balance and falls.

\section{Abbreviations}

PRISMA: Preferred reporting items for systematic reviews and meta-analysis; AE: Aquatic exercise; LE: Land exercise

\section{Acknowledgements}

The abstract of this study has been published on Medicine \& Science in Sports \& Exercise. 2020 July; 52(7S):143.

\section{Authors' contributions}

YK and EB: study conception and design. YK and MNV: data acquisition, screening, and coding. YK, MNV, and BW: risk of bias assessment and metaanalysis. YK: initial writing and drafting of the manuscript. All authors interpreted the data and revised critically for important intellectual content and approved the final version to be submitted.

\section{Funding}

Not declared.

\section{Availability of data and materials}

The datasets generated and analyzed during the current study are available from the corresponding author on reasonable request.

\section{Ethics approval and consent to participate}

Not applicable.

\section{Consent for publication}

Not Applicable.

\section{Competing interests}

The authors declare that they have no competing interests.

\section{Author details}

${ }^{1}$ Department of Kinesiology and Health Science, Utah State University, 7000 Old Main Hill, Logan, UT 84322-7000, USA. ²Department of Sport Science, Reykjavík University, Reykjavík, Iceland.

Received: 31 March 2020 Accepted: 12 August 2020

Published online: 25 August 2020

\section{References}

1. Bergen G. Falls and Fall Injuries Among Adults Aged $\geq 65$ Years United States, 2014. MMWR Morb Mortal Wkly Rep [Internet]. 2016 [cited 2019 Mar 30];65. Available from: https://www.cdc.gov/mmwr/ volumes/65/wr/mm6537a2.htm.

2. Stevens JA, Mack KA, Paulozzi LJ, Ballesteros MF. Self-reported falls and fallrelated injuries among persons aged $\geq 65$ years-United States, 2006. J Saf Res. 2008 Jan 1;39(3):345-9.

3. Alamgir $\mathrm{H}$, Muazzam S, Nasrullah M. Unintentional falls mortality among elderly in the United States: time for action. Injury. 2012 Dec 1;43(12): 2065-71.

4. Burns ER, Stevens JA, Lee R. The direct costs of fatal and non-fatal falls among older adults — United States. J Saf Res. 2016 Sep 1:58:99-103.

5. Sherrington C, Michaleff ZA, Fairhall N, Paul SS, Tiedemann A, Whitney J, et al. Exercise to prevent falls in older adults: an updated systematic review and meta-analysis. Br J Sports Med. 2017 Dec;51(24):1750-8.

6. Chan WC, Fai Yeung JW, Man Wong CS, Wa Lam LC, Chung KF, Hay Luk JK, et al. Efficacy of physical exercise in preventing falls in older adults with cognitive impairment: a systematic review and meta-analysis. J Am Med Dir Assoc. 2015 Feb 1:16(2):149-54

7. Okubo Y, Schoene D, Lord SR. Step training improves reaction time, gait and balance and reduces falls in older people: a systematic review and meta-analysis. Br J Sports Med. 2017 Apr 1;51(7):586-93.

8. Burton E, Cavalheri V, Adams R, Oakley Browne C, Bovery-Spencer P, Fenton $A M$, et al. Effectiveness of exercise programs to reduce falls in older people with dementia living in the community: a systematic review and metaanalysis. Clin Interv Aging. 2015 Feb 9:10:421-34.

9. Howe TE, Rochester L, Neil F, Skelton DA, Ballinger C. Exercise for improving balance in older people. Cochrane Database of Systematic Reviews [Internet]. 2011 [cited 2019 Mar 31];(11). Available from: https://www. cochranelibrary.com/cdsr/doi/10.1002/14651858.CD004963.pub3/full.

10. Rubenstein LZ. Falls in older people: epidemiology, risk factors and strategies for prevention. Age Ageing. 2006;35(suppl_2):ii37-41.

11. Arnold CM, Busch AJ, Schachter CL, Harrison EL, Olszynski WP. A Randomized Clinical Trial of Aquatic versus Land Exercise to Improve Balance, Function, and Quality of Life in Older Women with Osteoporosis. Physiotherapy Canada Physiotherapie Canada. 2008;60(4):296-306 2008 Fall.

12. Martínez-Carbonell Guillamón E, Burgess L, Immins T, Martínez-Almagro Andreo A, Wainwright TW. Does aquatic exercise improve commonly reported predisposing risk factors to falls within the elderly? A systematic review. BMC Geriatr. 2019 Feb 22;19(1):52

13. Waller B, Ogonowska-Słodownik A, Vitor M, Rodionova K, Lambeck J, Heinonen $A$, et al. The effect of aquatic exercise on physical functioning in the older adult: a systematic review with meta-analysis. Age Ageing. 2016 Sep 1;45(5):593-601.

14. Bressel E, Wing JE, Miller Al, Dolny DG. High-intensity interval training on an aquatic treadmill in adults with osteoarthritis: effect on pain, balance, function, and mobility. J Strength Conditioning Res. 2014 Aug; 28(8):2088-96.

15. Morris DM. Aquatic therapy to improve balance dysfunction in older adults. Topics Geriatr Rehabil. 2010 Apr;26(2):104-19.

16. Bressel E, Louder TJ, Dolny DG. Age-related changes in postural sway are not consistent between land and aquatic environments. J Geriatr Phys Ther 2017 Jul;40(3):113-20.

17. Batterham Sl, Heywood S, Keating JL. Systematic review and meta-analysis comparing land and aquatic exercise for people with hip or knee arthritis on function, mobility and other health outcomes. BMC Musculoskelet Disord. 2011 Jun 2;12(1):123.

18. Zampogna B, Papalia R, Papalia GF, Campi S, Vasta S, Vorini F, et al. The role of physical activity as conservative treatment for hip and knee osteoarthritis in older people: a systematic review and meta-analysis. J Clin Med. 2020 Apr;9(4):1167.

19. Winter DA, Patla AE, Frank JS. Assessment of balance control in humans. Med Prog Technol. 1990 May;16(1-2):31-51.

20. Frank JS, Patla AE. Balance and mobility challenges in older adults: Implications for preserving community mobility. Am J Prev Med. 2003;25(3, Supplement 2):157-63.

21. Louder TJ, Bressel E, Nardoni C, Dolny DG. Biomechanical comparison of loaded countermovement jumps performed on land and in water. Strength Cond Res. 2019 Jan;33(1):25-35.

22. Louder T, Bressel E, Baldwin M, Dolny DG, Gordin R, Miller A. Effect of aquatic immersion on static balance. Int J Aquatic Res Education. 2014 Feb;8(1):53-65.

23. Silvers WM, Bressel E, Dickin DC, Killgore G, Dolny DG. Lower-extremity muscle activity during aquatic and land treadmill running at the same speeds. J Sport Rehabil. 2014 May 1;23(2):107-22.

24. Sterne JAC, Savović J, Page MJ, Elbers RG, Blencowe NS, Boutron I, et al. RoB 2: a revised tool for assessing risk of bias in randomised trials. BMJ [Internet] 2019 Aug 28 [cited 2020 Jan 7];366. Available from: https://www.bmj.com/ content/366/bmj.14898

25. Sterne JAC, Gavaghan D, Egger M. Publication and related bias in metaanalysis: power of statistical tests and prevalence in the literature. J Clin Epidemiol. 2000 Nov 1;53(11):1119-29.

26. Sterne JAC, Sutton AJ, loannidis JPA, Terrin N, Jones DR, Lau J, et al. Recommendations for examining and interpreting funnel plot asymmetry in meta-analyses of randomised controlled trials. BMJ [Internet]. 2011;343 [cited 2020 Jun 2] Available from: https://www.bmj. com/content/343/bmj.d4002.

27. ICH Harmonised Tripartite Guideline. Statistical principles for clinical trials. International conference on harmonisation E9 expert working group. Stat Med. 1999 Aug 15;18(15):1905-42.

28. Shumway-Cook A, Woollacott MH. Postural Control. In: Motor Control: Translating Research Into Clinical Practice. Lippincott Williams \& Wilkins; 2016. p. 382-650.

29. Lesinski M, Hortobágyi T, Muehlbauer T, Gollhofer A, Granacher U. Effects of balance training on balance performance in healthy older adults: a systematic review and meta-analysis. Sports Med. 2015 Dec 1;45(12):1721-38. 
30. Adsett J, Morris N, Kuys S, Hwang R, Mullins R, Khatun M, et al. Aquatic exercise training is effective in maintaining exercise performance in trained heart failure patients: a randomised crossover pilot trial. Heart Lung Circ. 2017 Jun;26(6):572-9.

31. Cohen J. The t test for means. In: Statistical power analysis for the behavioral sciences. 2nd ed. Hillsdale, NJ: Lawrence Erlbaum Associates; 1988. p. 20-7.

32. Avelar NCP, Bastone AC, Alcântara MA, Gomes WF. Effectiveness of aquatic and non-aquatic lower limb muscle endurance training in the static and dynamic balance of elderly people. Revista Brasileira De Fisioterapia (Sao Carlos (Sao Paulo, Brazil)). 2010;14(3):229-36.

33. Simmons V, Hansen PD. Effectiveness of water exercise on postural mobility in the well elderly: an experimental study on balance enhancement. J Gerontol A Biol Sci Med Sci. 1996 Sep;51(5):M233-8.

34. Bergamin M, Ermolao A, Tolomio S, Berton L, Sergi G, Zaccaria M. Waterversus land-based exercise in elderly subjects: effects on physical performance and body composition. Clin Interv Aging. 2013;8:1109-17.

35. Zivi I, Maffia S, Ferrari V, Zarucchi A, Molatore K, Maestri R, et al. Effectiveness of aquatic versus land physiotherapy in the treatment of peripheral neuropathies: a randomized controlled trial. Clin Rehabil. 2018 May;32(5):663-70.

36. Pérez-de la Cruz S. A bicentric controlled study on the effects of aquatic $A$ Chi in Parkinson disease. Complement Ther Med. 2018;36:147-53.

37. Pérez de la Cruz S. Effectiveness of aquatic therapy for the control of pain and increased functionality in people with Parkinson's disease: a randomized clinical trial. Eur J Phys Rehabil Med. 2017 Dec;53(6):825-32.

38. Vivas J, Arias P, Cudeiro J. Aquatic therapy versus conventional land-based therapy for Parkinson's disease: an open-label pilot study. Arch Phys Med Rehabil. 2011 Aug;92(8):1202-10.

39. Volpe D, Giantin MG, Maestri R, Frazzitta G. Comparing the effects of hydrotherapy and land-based therapy on balance in patients with Parkinson's disease: a randomized controlled pilot study. Clin Rehabil. 2014 Dec;28(12):1210-7

40. Volpe D, Giantin MG, Manuela P, Filippetto C, Pelosin E, Abbruzzese G, et al. Water-based vs. non-water-based physiotherapy for rehabilitation of postural deformities in Parkinson's disease: a randomized controlled pilot study. Clin Rehabil. 2017 Aug;31(8):1107-15.

41. Conway ZJ, Blackmore T, Silburn PA, Cole MH. Dynamic balance control during stair negotiation for older adults and people with Parkinson disease. Hum Mov Sci. 2018 Jun 1;59:30-6.

42. Cauley JA. Osteoporosis: fracture epidemiology update 2016. Curr Opin Rheumatol. 2017:29(2):150-6.

43. Abreu DC, Trevisan DC, Costa GC, Vasconcelos FM, Gomes MM, Carneiro AA The association between osteoporosis and static balance in elderly women. Osteoporos Int. 2010 Sep 1;21(9):1487-91.

44. Liu-Ambrose T, Eng JJ, Khan KM, Carter ND, McKay HA. Older women with osteoporosis have increased postural sway and weaker quadriceps strength than counterparts with Normal bone mass: overlooked determinants of fracture risk? J Gerontol A Biol Sci Med Sci. 2003 Sep 1;58(9):M862-6.

45. Granacher U, Muehlbauer T, Gollhofer A, Kressig RW, Zahner L. An intergenerational approach in the promotion of balance and strength for fall prevention - a mini-review. GER. 2011;57(4):304-15.

46. Bosse I, Oberländer KD, Savelberg HH, Meijer K, Brüggemann G-P, Karamanidis K. Dynamic stability control in younger and older adults during stair descent. Hum Mov Sci. 2012 Dec 1;31(6):1560-70.

47. Schaefer SY, Louder TJ, Foster S, Bressel E. Effect of water immersion on dual-task performance: implications for aquatic therapy. Physiother Res Int. 2016 Sep;21(3):147-54

48. Louder T, Dolny D, Bressel E. Biomechanical comparison of countermovement jumps performed on land and in water: age effects. Sport Rehabil. 2018 May 1;27(3):249-56.

49. US Department of Health and Human Services. Physical Activity Guidelines for Americans. 2nd ed: US Department of Health and Human Services; 2018

50. Bressel E, Louder TJ, Hoover JP, Roberts LC, Dolny DG. Acute and chronic effects of aquatic treadmill training on land treadmill running kinematics: a cross-over and single-subject design approach. J Sports Sci. 2017 Nov 2; 35(21):2105-13.

51. Garner R, Wagner D, Bressel E, Dolny D. Land Versus Water Treadmil Running: Lactate Threshold. International Journal of Aquatic Research and Education [Internet]. 2014 Feb 1:8(1). Available from: https://scholarworks. bgsu.edu/ijare/vol8/iss1/3.
52. Bressel E, Smith G, Miller A, Dolny D. Aquatic-Treadmill Walking: Quantifying Drag Force and Energy Expenditure. Journal of Sport Rehabilitation [Internet]. 2012 Nov 1 [cited 2020 Feb 8];21(4). Available from: https:// journals.humankinetics.com/view/journals/jsr/21/4/article-jsr.2012.tr5.xml.xml.

53. Bressel E, Dolny DG, Gibbons M. Trunk muscle activity during exercises performed on land and in water. Med Sci Sports Exerc. 2011 Oct;43(10): 1927-32.

54. Denning W, Bressel E, Dolny D. Underwater Treadmill Exercise as a Potential Treatment for Adults With Osteoarthritis. International Journal of Aquatic Research and Education [Internet]. 2010 Feb 1;4(1). Available from: https:// scholarworks.bgsu.edu/ijare/vol4/iss1/9.

55. Song F, Hooper L, Loke YK. Publication bias: what is it? How do we measure it? How do we avoid it? Open Access Journal of Clinical Trials [Internet]. 2013 Jul 4 [cited 2020 Jul 9];5:71-81. Available from: https://www.dovepress. com/publication-bias-what-is-it-how-do-we-measure-it-how-do-we-avoid-itpeer-reviewed-article-OAJCT.

\section{Publisher's Note}

Springer Nature remains neutral with regard to jurisdictional claims in published maps and institutional affiliations.
Ready to submit your research? Choose BMC and benefit from:

- fast, convenient online submission

- thorough peer review by experienced researchers in your field

- rapid publication on acceptance

- support for research data, including large and complex data types

- gold Open Access which fosters wider collaboration and increased citations

- maximum visibility for your research: over $100 \mathrm{M}$ website views per year

At BMC, research is always in progress.

Learn more biomedcentral.com/submissions 